\title{
Anterior Mediastinal Teratoma- A Rare Variety
}

Palash SMAZN 1 , Hossain MD², Khan TMNS ${ }^{3}$, Meher T4 ${ }^{4}$, Hasan $\mathrm{MK}^{5}$, Khan $\mathrm{SI}^{6}$, Ahmed N Ahmed $\mathrm{S}^{8}$, Haider $\mathrm{MZ}^{9}$

\begin{abstract}
Anterior mediastinal teratomas are rare germ cell tumors. We report a case of such rare tumor in a 16 years old boy who presented with sudden severe right sided abdominal pain which radiating to chest for one day. CT scan of the chest was suggestive of anterior mediastinal teratoma. Patient underwent Clamshell thoracotomy. Peroperatively a large mass was found in the anterior mediastinum containing huge amount of sebum like material. It was adherent to mediastinal surface of right \& left lung, pericardium and great vessels (SVC, Aorta). The tumor and was resected out except part of its posterior surface which was adherent to pericardium and great vessels and was left in situ. He had a smooth \& uneventful post-operative recovery. Histopathology reported as mature (benign) teratoma with no evidence of malignancy.
\end{abstract}

\section{Keywords}

Mediastinal Teratoma. Extra-gonadal germ cell tumor

\section{Introduction}

Germ cell tumors are predominantly found in the gonads, and the most common extragonadal site is the anterior mediastinum. ${ }^{1}$ Mediastinal germ cell tumors account for $15 \%$ of all mediastinal tumors in adults and $24 \%$ in children. ${ }^{2}$ Of the tumors of the anterior mediastinum, benign cystic teratomas have excellent prognosis after complete surgical excision.

\section{Case Report}

A 16-year-old non-diabetic, normotensive boy came to Emergency Room of Apollo Hospitals with the complaints of sudden severe right upper abdominal pain radiating to the chest associated with shortness of breath for 1 day. He has no history of cough, hemoptysis, heaviness of chest, fever anorexia, weight loss. After having treatment in local hospital with loading dose of aspirin he was brought to this hospital. At ER, clinically he was not anemic, having tachycardia, tachypnea with increased BP. His abdomen was soft, non-tender, having no organomegaly, percussion note was diminished on upper and mid-zone of left lung and breath sound was also diminished in the same area. Heart sound was normal. ECG showed sinus tachycardia. Chest X-ray (Fig. 1) shows a soft tissue shadow occupying mediastinum, medial side of right lung and most of the left lung field. Abdominal x-ray

1. Specialist, Dept. of Cardiovascular and Thoracic Surgery, Apollo Hospitals Dhaka.2. Senior registrar, Dept. of Cardiothoracic \& Vascular Surgery, Apollo Hospitals Dhaka. 3. Registrar, Dept. of Cardiothoracic \& Vascular Surgery, Apollo Hospitals Dhaka.4.Registrar Dept. of Cardiothoracic \& Vascular Surgery, Apollo Hospitals Dhaka. 5.Registrar, Dept. of Cardiothoracic \& Vascular Surgery, Apollo Hospitals Dhaka. 6. Specialist, Dept. of Cardiothoracic Anesthesia, Apollo Hospitals Dhaka 7.Consultant,Dept. of Cardiothoracic Anesthesia, Apollo Hospitals Dhaka 8. Consultant,Dept. of Cardiovascular and Thoracic Surgery,Apollo Hospitals Dhaka.9. Consultant,Department of Cardiovascular and Thoracic Surgery,Apollo Hospitals Dhaka. 
Abdominal x-ray was normal. Chest Ultrasonogram: Right sided mild pleural effusion (approximate volume $65-110 \mathrm{ml}$ ) with basal consolidation.

CT Chest showed Large lobulated, multilocular anterior mediastinal mass (Fig. 2\&3) measuring $17 \mathrm{~cm}$ (transverse) $\mathrm{x} 8.5 \mathrm{~cm}$ (anteroposterior) $\mathrm{x}$ $9.5 \mathrm{~cm}$ (craniocaudal) in size.

There was peripheral rim calcifications and intralesional fluid attenuation and macroscopic fat densities. The fat planes of the mass with mediastinal vascular structures are largely preserved; however, the fat planes with the

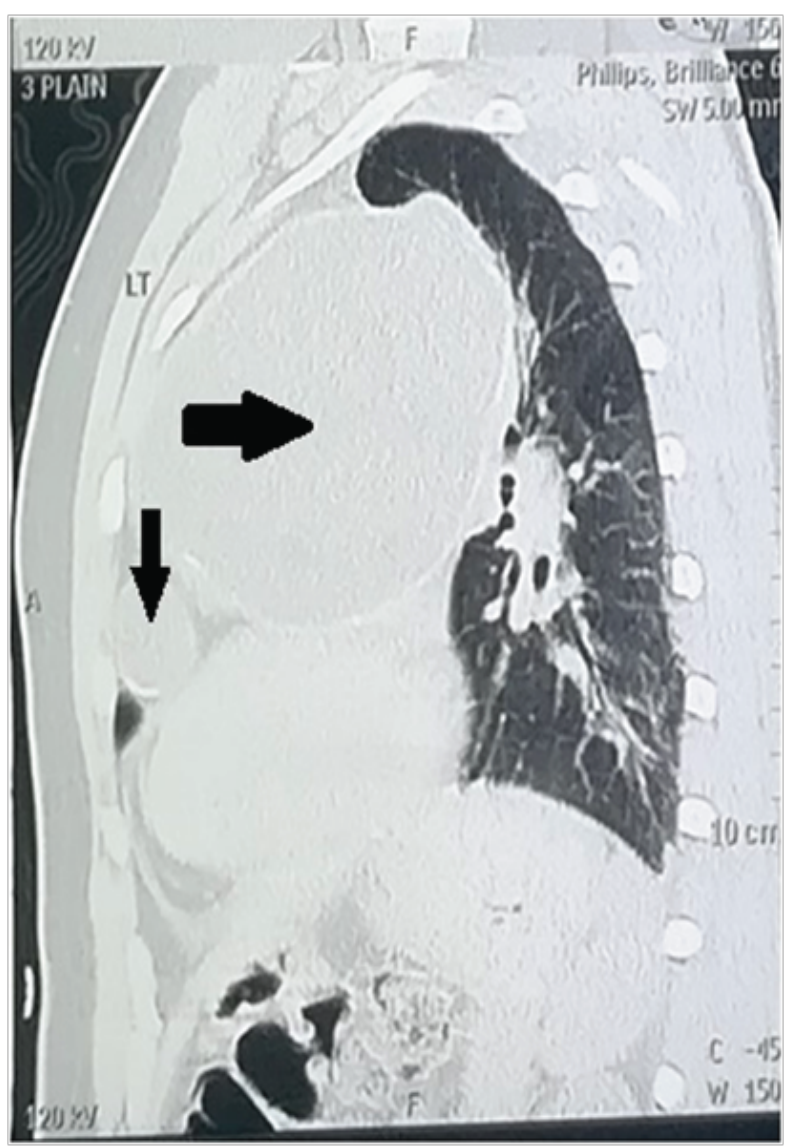

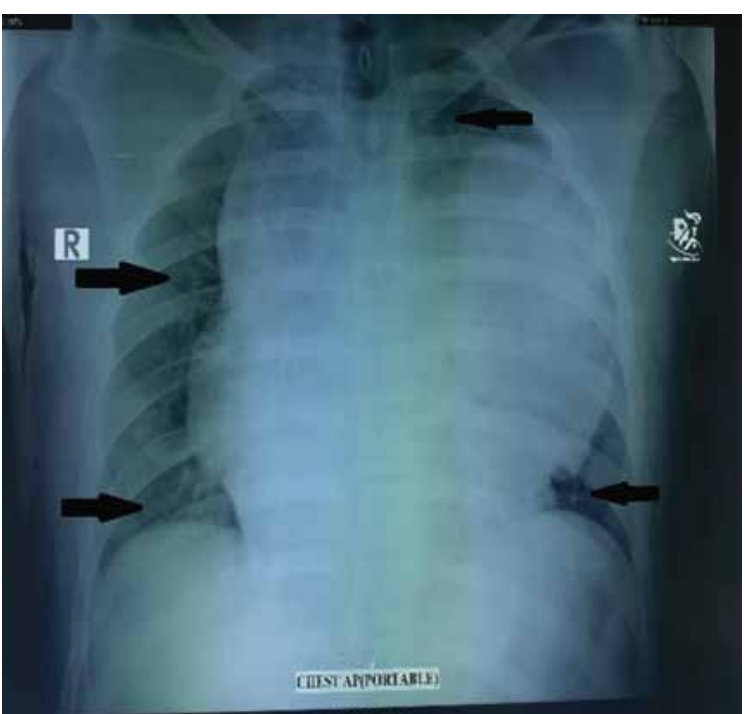

Fig. 1: Shows a soft tissue shadow occupying mediastinum, medial side of right lung ( $\rightarrow$ Arrows) and most of the left lung field ( $\leftarrow$ Arrows).
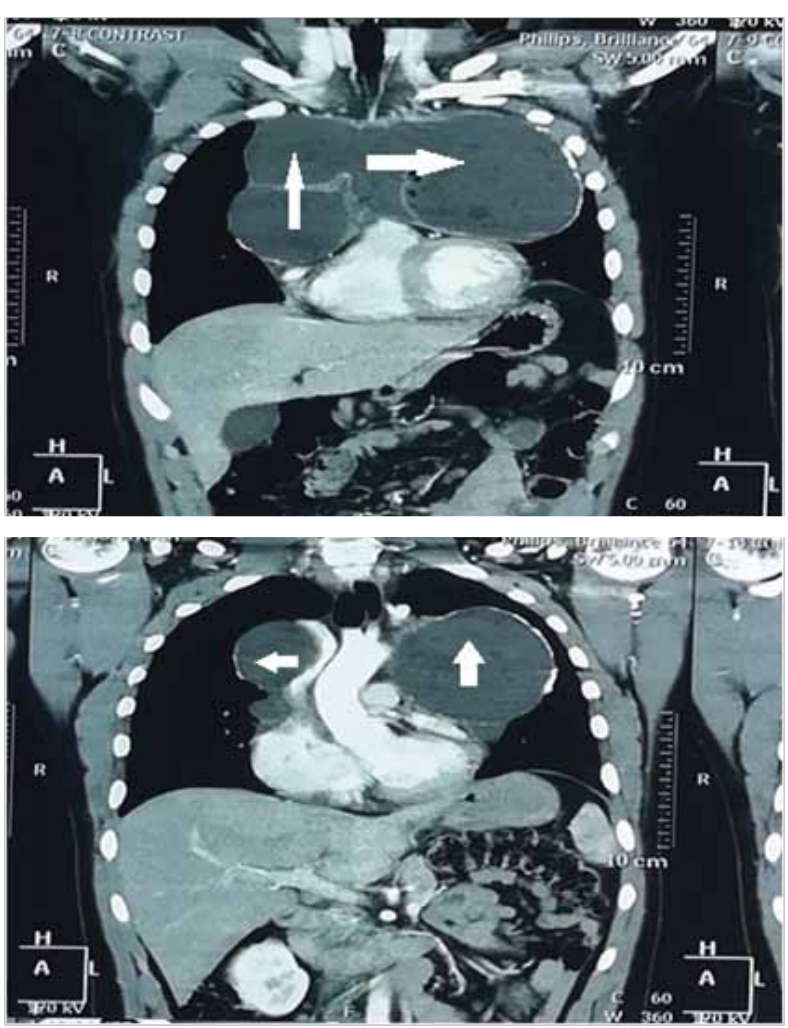

Fig. 2\&3: CT Chest showing large lobulated, multilocular anterior mediastinal mass (Black \& White Arrows) 
pericardium is indistinct at places. No suggestion of chest wall invasion was seen. Trachea and major bronchi are normal. Mediastinal vascular structures and heart otherwise appear normal. No significant mediastinal or hilar lymphadenopathy seen. Moderate right sided pleural effusion with right posterior basal segmental consolidation and basal lung atelectasis CT scan impression was large anterior mediastinal mass? Thymic/ Teratoma/ Multilocularthymic cyst with Teratoma component with right pleural effusion with basal lung consolidation and atelectasis. CT guided FNAC revealed Fairly cellular smears show acute and chronic inflammatory cells

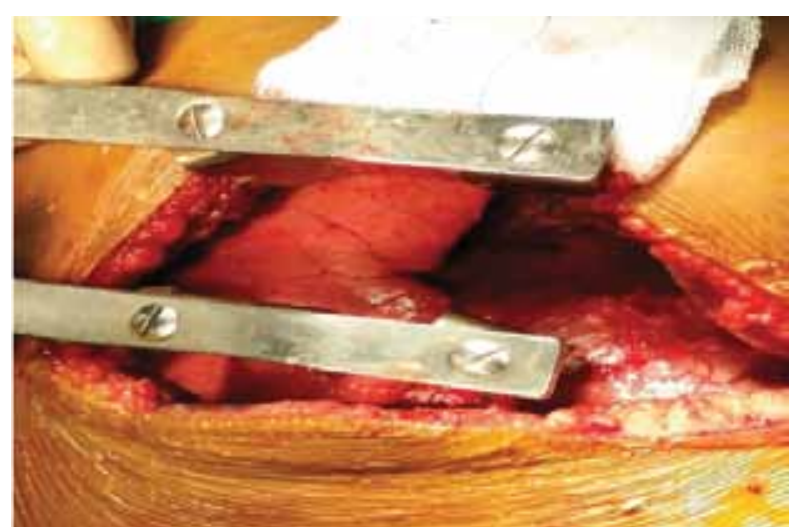

Fig. 4:Thoracotomy by Clamshell incision

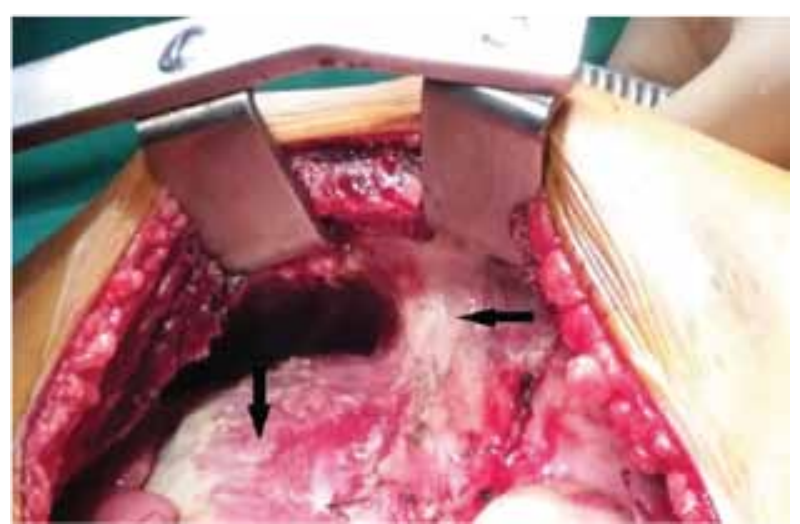

Fig. 5: Large mediastinal mass adherent to adjacent structures \& posterior surface of sternum(Arrows). admixed with foamy macrophages and clusters of regular squamous cells in a proteinaceous background. No evidence of granuloma or atypical cell is seen. Compatible with infected mediastinal cyst. The patient was planned for complete resection. Under G/A with double lumen endotracheal tube with all aseptic precaution Clamshell incision (Fig. 4) given.

A large cystic mediastinal mass (Fig. 5) was found which was adherent with upper part of mediastinal surface of right lung, upper and middle part of mediastinal surface of left lung and upper part of anterior surface of pericardium and great vessels (SVC, Aorta). Laterally it was adherent to phrenic nerve. Anterior surface of the mass could be separated from posterior surface of the sternum, and lateral surface could be separated from right and left lung, but separation from anterior surface of pericardium and great vessel was not possible as it was tightly adherent to those areas. So the tumor was perforated at multiple sites and huge amount of sebum like material (Fig. 6) was removed from multiple segment. After removal of all sebaceous materials, the tumor was resected out except part of posterior surface of the mass which was adherent to pericardium and great vessels which was left in situ.

Resected part (Fig. 7) of the mass was sent for histopathological examination. After achieving haemostasis and thorough mediastinal toileting chest was closed keeping two drains in both pleural cavities. Serial postoperative X-rays showed good right lung expansion (Fig. 8). 

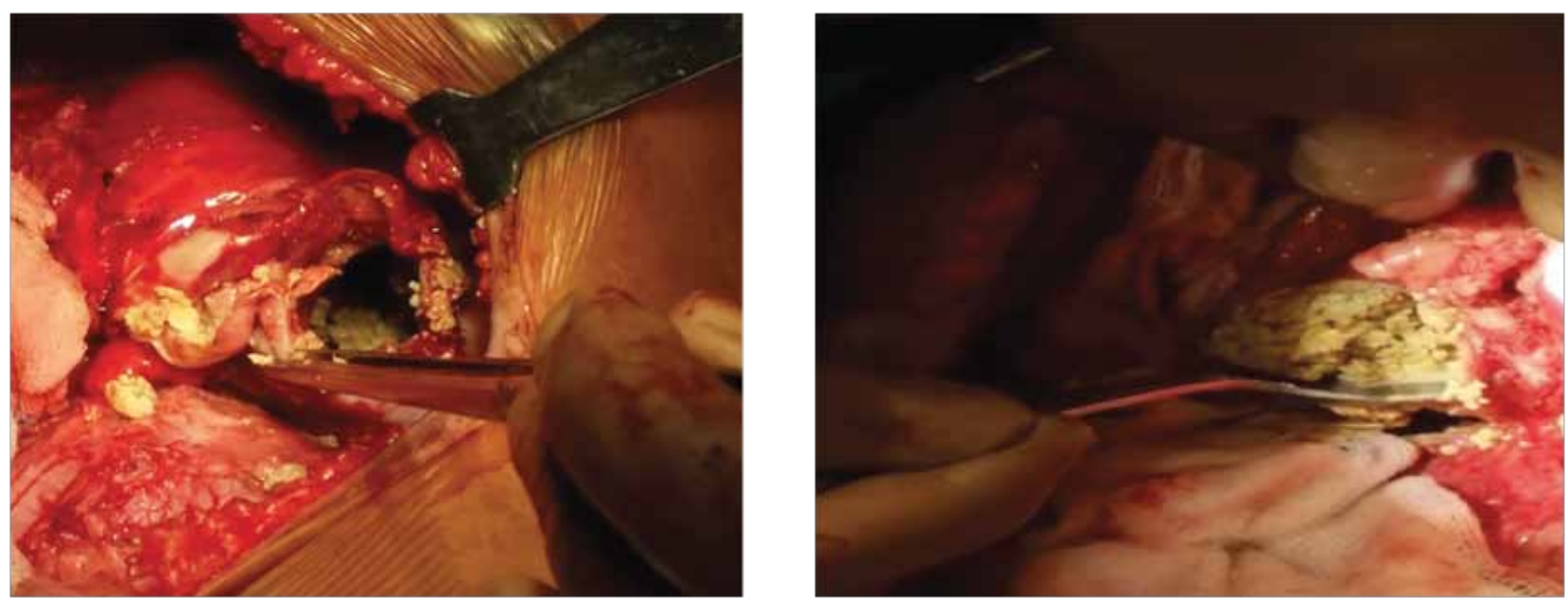

Fig. 6 (a \&b): Tumour containing huge amount of sebum like material

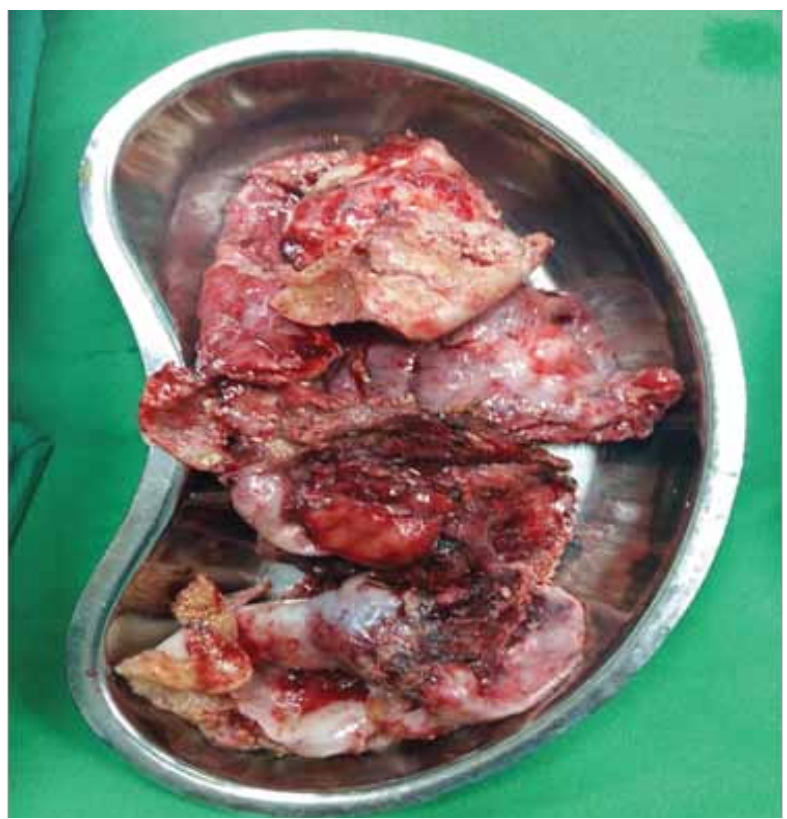

Fig. 7: The tumor was removed in multiple segments and sent for histopathology

In histopathology, sections from the submitted specimen reveal the features consistent with mature teratoma. No evidence of malignancy is seen. His postoperative course was uneventful and was discharge to home on 9th post-op day. On subsequent visits in OPD till date he was found to be recovered well without any significant complication and no evidence of recurrence.

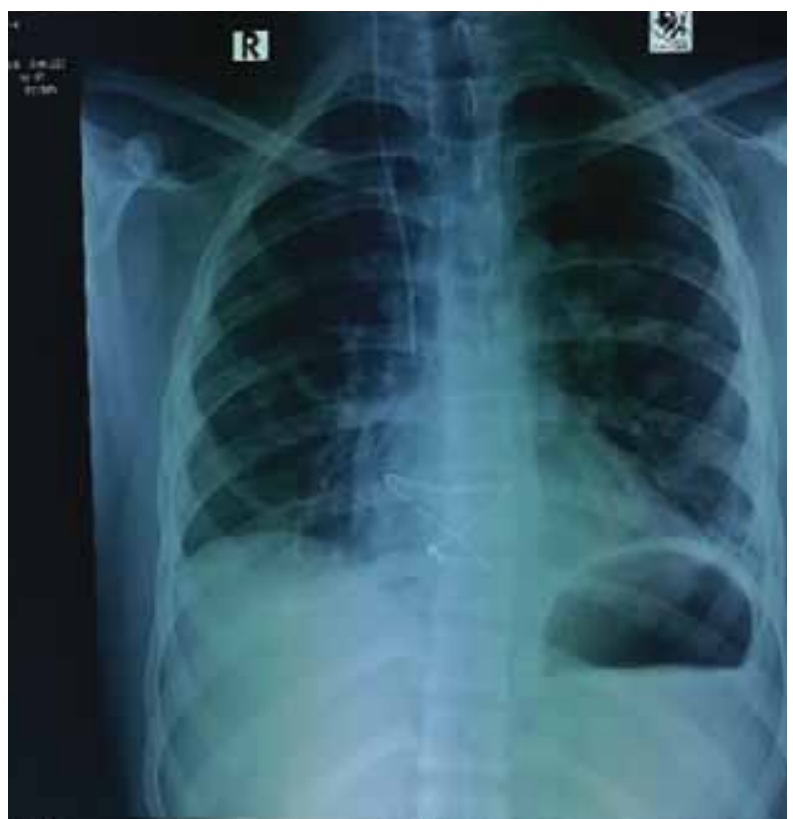

Fig. 8: Postoperative X-rays showed good right lung expansion

recurrence. However as small part of the tumor was left in situ so there is chance of recurrence so he is advised to follow up in OPD on regular basis for at least 2 years.

\section{Discussion}

Germ cell tumors are uncommon neoplasms that usually arise in the gonads. The most common 


\section{CASE REPORT}

extragonadal site is anterior mediastinum. It is estimated that only $1-3 \%$ of all germ cell tumors arise in the mediastinum. ${ }^{1}$ Germ cell tumors account for $15 \%$ of adult anterior mediastinal tumors and $24 \%$ of all pediatric anterior mediastinal tumors. Teratomas are equally present in men and women with an age range from 1 to 73 years, and average age at presentation is 28 years. There is now general acceptance that extra gonadal germ cell tumors represent malignant transformation of germ cell elements within these sites without a gonadal primary focus. A theory to account for extragonadal germ cell tumors was proposed by Fine $^{3}$, who suggested that there was an error immigration of primitive germ cells along the urogenital ridge. Histologically mediastinal germ cell tumors are classified as teratomas (which may be mature or immature), teratoma with malignant elements, teratocarcinoma, seminoma, and nonseminomatous tumors. Benign teratomas are often asymptomatic and are discovered on chest radiograph obtained for unrelated reasons. If symptoms are present, they are due to mass effect and may cause cough, dyspnea, or chest pain. Teratomas may rarely cause superior vena cava syndrome. Rarely the patient may have expectoration of hair (trichoptysis) and is pathognomonic symptom. CT with IV contrast is the imaging modality of choice with a suspected germ cell tumor. Benign teratomas are usually rounded with sharp margins. They often contain variable amount of fat, soft tissue density, cystic areas, calcification, and bone or teeth. A fat fluid level may be seen in cystic teratomas. Complete resection is curative for a benign teratoma, and there is no role for adjuvant chemotherapy or radiotherapy. ${ }^{4}$

\section{Conclusion}

Even though anterior mediastinal teratomas are uncommon tumors, complete excision of the tumor without any surgical complication is possible in most of the cases. Hence, these tumors can be cured by surgical excision.

\section{References}

1. Nichols CR. Mediastinal germ cell tumors: clinical features and biologic correlates. Chest. 1999; 99(2):472-9.

2. Mulen B, Richardson JD. Primary anterior mediastinal tumors in children and adults. Ann ThoracSurg. 1986; 42:338-45.

3. Fine G, Smith RW Jr, Pachter MR. Primary extragenit al choriocarcinoma in the male subject. Case report and review of literature. Am J Med.1962; 32:776-94.

4. Saabye J, Elbrik A, Anderson K. Teratomas of the anterior mediastinum. Scand J ThoracCardiovascSurg.1987; 21(3): 271-2. 